\title{
Involvement of mitochondrial-targeted RecA in the repair of mitochondrial DNA in the moss, Physcomitrella patens
}

\author{
Masaki Odahara $^{1}$, Takayuki Inouye ${ }^{1}$, Tomomichi Fujita ${ }^{2 \dagger}$, \\ Mitsuyasu Hasebe ${ }^{2,3}$ and Yasuhiko Sekine ${ }^{1 *}$ \\ ${ }^{1}$ Department of Life Science, College of Science, Rikkyo (St. Paul's) University, 3-34-1, \\ Nishi-Ikebukuro, Toshima-ku, Tokyo 171-8501, Japan \\ ${ }^{2}$ National Institute for Basic Biology, Okazaki 444-8585, Japan \\ ${ }^{3}$ Department of Basic Biology, The Graduate University for Advanced Studies, \\ Okazaki 444-8585, Japan
}

(Received 24 November 2006, accepted 29 December 2006)

\begin{abstract}
Homologous recombination is a universal process that contributes to genetic diversity and genomic integrity. Bacterial-type RecA generally exists in all bacteria and plays a crucial role in homologous recombination. Although RecA homologues also exist in plant mitochondria, there have been few reports about the in vivo functions of these homologues. We identified a recA gene orthologue (named PprecA1) in a cDNA library of the moss, Physcomitrella patens. N-terminal fusion of the putative organellar targeting sequence of PpRecA1 to GFP caused a targeting of PpRecA1 to mitochondria. PprecA1 partially complemented the effects of a DNA damaging agent in an Escherichia coli recA deficient strain. Additionally, the expression of PprecA1 was induced by treating the plants with DNA damaging agents. Disruption of PprecA1 by targeted replacement resulted lower rate of the recovery of the mitochondrial DNA from methyl methan sulfonate damage. This is the first report about the characteristics of a null mutant of bacterial-type $\operatorname{rec} A$ gene in plant. The data suggest that PprecA1 participates in the repair of mitochondrial DNA in P. patens.
\end{abstract}

Key words: mitochondria, RecA, DNA repair, Physcomitrella patens

\section{INTRODUCTION}

Homologous recombination is a universal process that occurs in all organisms. It is responsible for genetic diversity, the maintenance of genomic integrity, and the proper segregation of chromosomes (Kowalczykowski et al., 1994). The molecular mechanism of homologous recombination has been well studied in Escherichia coli by extensive genetic and molecular analyses. RecA protein is a crucial component in homologous recombination (Roca and Cox, 1997). It binds to DNA to form a nucleoprotein filament that then aligns with a homologous duplex to promote single strand exchange (Lusetti and Cox, 2002). Recent studies suggest that RecA and other recombination proteins also function in the nonmutagenic repair of stalled or collapsed replication forks (Lusetti and Cox, 2002).

\footnotetext{
Edited by Hisaji Maki

* Corresponding author. E-mail: ysekine@rikkyo.ac.jp

$\dagger$ Present address: Graduate School of Science, Hokkaido University, Kita 10 Nishi 8, Sapporo 060-0810, Japan
}

Homologues of RecA have been identified in many prokaryotes and eukaryotes (Lin et al., 2006). In eukaryotes, there are several RecA homologues, including Rad51 (Shinohara et al., 1992) and Dmc1 (Bishop et al., 1992), for homologous recombination in the nuclear genome. In addition, a plant Arabidopsis thaliana gene encoding a RecA homologue, which is distinct from Rad51/Dmc1 and closely related to bacterial RecA proteins, has been characterized and found to be targeted to chloroplasts (At1g79050 [AtcprecA]; Cerutti et al., 1992, Cao et al., 1997). RecA-like strand transfer activity was also detected in a stromal extract from pea chloroplast (Cerutti and Jagendorf, 1993). The complete nuclear genome sequence of A. thaliana (The Arabidopsis Genome Initiative, 2000) revealed the presence of two other recA genes (At3g10140 [AtmtrecA] and At2g19490) related to bacterial recA, and At3g10140 was reported to be targeted to mitochondria (Khazi et al., 2003). Strand transfer activity and recombination intermediates were reported in soybean mitochondria (Manchekar et al., 2006); however, the biological significance of homologous recombina- 
tion in plant mitochondria and the in vivo role of bacterial-type RecA have not been addressed.

Mitochondria in plants play an essential role in the cell as the major producers of ATP via oxidative phosphorylation. The majority of genes from the original bacterial endosymbiont have been lost or transferred to the nucleus in the course of evolution (Palmer et al., 2000). Therefore, mitochondrial genomes encode only a small number of RNAs and proteins, including ribosomal RNAs, transfer RNAs, some subunits of the respiratory chain complexes, and a few ribosomal proteins (Unseld et al., 1997). Most of reported mitochondrial mutations result in defects in plant growth and development (Newton et al., 2004), indicating that mitochondrial genes are essential for the normal functioning of plant mitochondria.

The genome sequence of the moss, Physcomitella patens, in which gene targeting occurs with an efficiency similar to that observed in yeast (Schaefer and Zryd, 2001) has been nearing completely sequenced (Cove et al., 2006), opening the possibility of doing advanced molecular biological analysis in this organism. In this report, we demonstrate that the $P$. patens RecA homologue (PpRecA1) is specifically localized to mitochondria. To uncover the in vivo functions of this mitochondrial RecA homologue, we inactivated PprecA1 by targeted replacement. Investigation of the recovery from DNA damage in the resulting disruptant suggested involvement of PpRecA1 in the repair of mitochondrial DNA (mtDNA).

\section{MATERIALS AND METHODS}

Plant material and growth conditions Protonemata of Physcomitrella patens Bruch \& Schimp subsp. patens were cultured on cellophane-covered BCDATG medium [1 $\mathrm{mM} \mathrm{MgSO}_{4}, 10 \mathrm{mM} \mathrm{KNO}, 45 \mu \mathrm{M} \mathrm{FeSO}, 1.8 \mathrm{mM}$ $\mathrm{KH}_{2} \mathrm{PO}_{4}, 1 \mathrm{mM} \mathrm{CaCl}, 5 \mathrm{mM}$ ammonium tartrate, $0.5 \%$ (w/v) glucose, and $0.8 \%$ agar (Nishiyama et al., 2000)] or BCDAT liquid medium [1 $\mathrm{mM} \mathrm{MgSO}_{4}, 10 \mathrm{mM} \mathrm{KNO}{ }_{3}, 45$ $\mu \mathrm{M} \mathrm{FeSO}_{4}, 1.8 \mathrm{mM} \mathrm{KH} \mathrm{PO}_{4}, 1 \mathrm{mM} \mathrm{CaCl}, 5 \mathrm{mM}$ ammonium tartrate]. Protonemata were cultivated at $25^{\circ} \mathrm{C}$ under constant light conditions $\left(70 \mu \mathrm{mol} \mathrm{m} \mathrm{m}^{-2} \mathrm{~s}^{-1}\right)$.

Sequence and phylogenetic analysis of $\mathrm{rec} A$ genes The putative recA genes in A. thaliana and Oryza sativa genomes were retrieved from The Arabidopsis Information Resource (http://www.arabidopsis.org/) and TIGR Rice Genome Annotation databases (http://www.tigr.org/tdb/ e2k1/osa1/), respectively. The Cyanidioschyzon merolae recA gene was identified by a BLAST search of Cyanidioschyzon merolae Genome Project database (http://merolae.biol.s.u-tokyo.ac.jp/, Matsuzaki et al., 2004). Sequence alignment was performed by using the CLUSTALW multiple alignment program (Thompson et al., 1994). Evolutionary distance was calculated with the Kimura-2parameter and phylogenetic tree was constructed with the neighbor joining method (Saitou and Nei, 1987). Targeting prediction for proteins was performed with the 130 $\mathrm{N}$-terminal residues of them by TargetP (Emanuelsson et al., 2000). The accession numbers are E. coli RecA [AP_003266], Rickettsia prowazekii RecA [NP_221113], Synechocystis sp. PCC 6803 RecA [NP_443045], and Chlamydomonas reinhardtii RecA [AB048829]. The sequences of the full-length cDNAs corresponding to PprecA1 and PprecA2 were confirmed, annotated and submitted to the GenBank database (under Accession No AB284534 and AB284535, respectively). The genomic sequence of PprecA1 was amplified by PCR with the primers 5'-CCCTGATCAGGTACCCCCGGGGACATGGCGGGAGCTCTGCGAGTACTTAG-3' and 5'-CCCAAGCTTCACCATGGGATCCCCTGTCACCTCTAGATTATCAGCATC -3 , and sequenced.

Extraction of DNA and RNA Genomic DNA was extracted from protonemata cultivated four days after inoculation on agar plates by the cetyltrimethylammonium bromide method (Murray and Thompson, 1980). Total RNA was extracted from the protonemata using the RNeasy Plant Mini Kit (Qiagen, Hilden, Germany), and subsequently DNaseI treatment was performed to remove contaminating genomic DNA.

Moss transformation Isolation of protoplasts and subsequent PEG-mediated transformation of $P$. patens were performed according to Nishiyama et al. (2000). To generate stable transformants, $10 \mu \mathrm{g}$ of linearized targeting construct was introduced, and the resulting transformants were subsequently selected in medium containing $30 \mu \mathrm{g} / \mathrm{ml}$ hygromycin $\mathrm{B}$, according to Nishiyama et al. (2000).

Fluorescence microscopy The first 210 bp of PprecA1 cDNA was amplified by PCR with the primers (restriction sites are underlined): 5'-CCCTGATCAGGTACCCCCGGGGACATGGCGGGAGCTCTGCGAGTACTTAG-3' and 5'CCCAAGCTTCACCATGGCATTGGGGTTGTCAGGGTC G-3'. The PCR product was cloned into BamHI - NcoI cleaved p7133TP-sGFP (kindly provided by S. Aoki; Hara et al., 2001a) carrying a modified cauliflower mosaic virus 35S promoter E7133 (Mitsuhara et al., 1996). The construct was introduced into $P$. patens protoplasts, and one day after transformation, the GFP and chlorophyll fluorescence of the protoplasts were observed by fluorescence microscopy. For visualizing mitochondria, P. patens protoplasts were incubated in a medium containing $200 \mathrm{nM}$ Mito Tracker Green FM (Molecular Probes, Eugene, OR, USA.) or $500 \mathrm{nM}$ Mito Tracker Orange (Molecular Probes, Eugene, OR, USA.) for $45 \mathrm{~min}$ at $25^{\circ} \mathrm{C}$. Protoplasts were observed with an epifluorescence microscope (AX80; Olympus, Tokyo). 
Construction of the PprecA1 disruption vector The $1.1-\mathrm{kb}$ upstream region and 1.9-kb downstream region of genomic PprecA1 were amplified by PCR using the following primers; 5'-GGGATCGATGAGCTCGTCAACTTCACTTCTTAGAAC-3' and 5'-GTTCGGGAGCGATTAGTGCAGAAGC-3' for amplification of the 1.1-kb fragment, and 5'GGGTCTAGAGCATGGGTGACTAAGCAGC-3' and 5'GGGGAGCTCCCATAGTAAGTTGTTAGGTG-3' for amplification of the $1.9-\mathrm{kb}$ fragment. The resulting $1.1-\mathrm{kb}$ fragment cleaved by $\mathrm{Cla \textrm {I }}$ and the 1.9 -kb fragment cleaved by $X b a \mathrm{I}$ and $S a c \mathrm{I}$ were inserted into the $C l a \mathrm{I}$ site and between the $X b a \mathrm{I}$ and $S a c I$ sites of the hygromycin phosphotransferase (hpt) gene expression cassette of pTN186 (kindly provided by T. Nishiyama), respectively. The resulting plasmid containing the targeting construct was named pMAK151 and linearized before introducing it into $P$. patens protoploasts.

DNA gel blot analysis Total genomic DNA $(4 \mu \mathrm{g})$ was separated on a $0.8 \%$ agarose gel and blotted onto a nylon membrane. To prepare the probes, the upstream sequence of PprecA1 (1.1-kb fragment) and downstream sequence of PprecA1 (1.9 kb fragment) were excised from pMAK151, and randomly labeled with the DIG DNA labeling mix (Roche). Hybridization of the probes was performed at $37^{\circ} \mathrm{C}$ and the membranes were washed in 2 $\times \mathrm{SSC}$ with $0.1 \% \mathrm{SDS}$ at $25^{\circ} \mathrm{C}$ and $0.5 \times \mathrm{SSC}$ with $0.1 \%$ SDS at $65^{\circ} \mathrm{C}$. Detection of DIG-labeled probes was performed with Anti-DIG-alkaline phosphatase (Roche) and AttoPhos (Promega).

Semi-quantitative reverse transciptase-PCR (RTPCR) for expression analysis Reverse transcription was performed by using $1 \mu \mathrm{g}$ of total RNA and an oligo (dT) primer, after which PCR was performed. To amplify a segment of PprecA1, the primers 5'GCGTCTAGATTCACGTGATTGCACAGG-3' and 5'-CCCAAGCTTCACCATGGGATCCCCTGTCACCTCTAGATTATCAGCATC-3' were used in Fig. 4, and 5'-GGGAAGCTTCGGGTAGTGGAGATCTTTGG-3' and 5'-GGTGGTGATACTTTGTTC-3' were used in Fig. 5. To obtain internal controls, the primers 5'CATGTTCGAGACGTTCAACGTGCCG-3' and 5'-GATGGACCAGATTCATCGTACTCGC-3' were used to amplify a segment of the actin gene (Hara et al., 2001b) and the primers 5'CATGGTGGGATCGGCTAAG-3' and 5'TAATACGACTCACTATAGGGCGAGATAGGAGCATCTGTACC -3' were used to amplify a segment of the GAPDH gene (Leech et al., 1993). In these experiments, PCR amplification was performed in the exponential amplification phase.

For expression analysis of PprecA1 in methyl methane sulfonate (MMS)- or bleomycin-damaged cells, protonemata were cultivated in BCDAT liquid medium containing $20 \mathrm{mM}$ of MMS or $25 \mu \mathrm{g} / \mathrm{ml}$ of bleomycin for $6 \mathrm{~h}$ (under vacuum for the first $30 \mathrm{~min}$ ), and then total RNA was extracted.

Assay for complementation of $E$. coli recA deficient strain E. coli recA gene was amplified by PCR with the primers 5'-CCCTCATGATTATCGACGAAAACAAACAGAAAG-3' and 5'-CCCAAGCTTAAAAATCTTCGTTAGTTTCTGC-3', and the PCR product was cloned between the NcoI and HindIII sites of pBAD24 (Guzman et al., 1995), yielding pMAK132. The PprecA1 gene was amplified by PCR with the primers 5'-GGGCCATGGCTCTGGAGACTGTTACC-3' and 5'-GCCGGTCGACCTCCTACCCTGTCACCTC-3', and the PCR product was cloned between the NcoI and SalI sites of pBAD24, yielding pMAK131. E. coli CSR603 [recA1, uvrA6, phr-1] (Sancar and Rupert, 1978) cells bearing pMAK132 or pMAK131 were cultured to $\mathrm{OD}_{660}$ of 0.5 in M9 medium containing $0.2 \%$ glucose and then transferred to M9 medium containing $0.2 \%$ arabinose and incubated for $30 \mathrm{~min}$ at $37^{\circ} \mathrm{C}$ for gene induction. Cells were treated with different concentration of mitomycin $\mathrm{C}$ and incubated for $30 \mathrm{~min}$ at $37^{\circ} \mathrm{C}$. After washing, appropriate dilutions of treated cells were spread on LB plate and incubated overnight at $37^{\circ} \mathrm{C}$. Colony forming units (cfu) were counted, and the surviving fraction of cells was calculated by dividing the cfu in treated cells by the cfu in untreated cells.

PCR assay for monitoring recovery of DNA from MMS damage Protonemata of the wild type (WT) and PprecA1 disruptant were cultivated under vacuum in BCDAT liquid medium containing $20 \mathrm{mM}$ of MMS for $1 \mathrm{~h}$, and were then washed and transferred to BCDATG agar medium. The protonemata were subsequently cultivated and harvested at $0,3,6,12,24,48,72 \mathrm{~h}$ after washing. As a control for undamaged DNA, protonemata were harvested just before the MMS treatment. Total genomic DNA was extracted from the harvested cells and quantitated by using a Hoechst 33258 (Sigma) and DQ200 Dyna Quant 200 Fluorometer (GE Healthcare), according to the manufacturer's protocol. As a template for PCR, $10 \mathrm{ng}$ of total genomic DNA was used. To detect damage to the template DNA, PCR was performed to amplify cox2 $(3.2 \mathrm{~kb})$ with the primers 5'-TGTGGGGCCTGTTGAAGGGCTGAA3' and 5'-AGAGTGGGCTCAGCAGTCGGCCTA-3' for 18 cycles, $\operatorname{nad} 7(3.1 \mathrm{~kb})$ with the primers 5'-GAAGTTGTAGAACGCGCGGAACCGC-3' and 5'TAATACGACTCACTATAGGGCGAGTAGTTCTATCTATCTACCTCTCC-3' for 18 cycles, atp $9(3.8 \mathrm{~kb})$ with the primers 5'-GATCAGCCCTTGAATGGTCATTGT-3' and 5'-GCGCAGCCCTATTGGCTTTGCGAAT-3' for 18 cycles, PpMADS2 (3.9 kb; Henschel et al. 2002) with the primers 5'-GGACGTTGAAGGCACGGATTCTGTAGTG-3' and 5'CATCCTCATCAGGGGCCAAGTCATGTATC-3' for 26 cycles, GAPDH (3.0 kb) with the primers 5'CTTCCAGATCTAAGAGGAAGGACG-3' and 5'GATGACCGTAACATGAACGCTGGCG-3' for 26 cycles, tubG (4.0 kb) with the primers 5'-CCCTTTCCCTTTAGC- 
GATTCTGAG-3' and 5'-CCTGAAACCACATTGCCAAGGCGC-3' for 26 cycles. The PCR products were analyzed by electrophoresis in an agarose gel and the gel was stained with SYBR Green I (TakaRa). The integrated intensities of the product bands were determined by using Typhoon 9210 and Image Quant (GE Healthcare). In order to measure the effect of DNA damage on amplification, the intensity of the amplified product from damaged genomic DNA was divided by that from undamaged genomic DNA.

\section{RESULTS}

Identification of Physcomitrella patens recA Two kinds of distinct cDNA sequence encoding a putative recA gene homologue were identified by a BLAST search of full-length enriched cDNA libraries of $P$. patens (Nishiyama et al., 2003), and were designated PprecA1 and PprecA2, respectively. PprecA1 had one putative translation initiation site in its 5'region, and would encode a 435-residue protein. Except for an N-terminal peptide, which was predicted to be a mitochondrial presequence, the putative gene product shared high similarity with $E$. coli RecA protein and each of the A.thaliana RecA proteins (Fig. 1A). PpRecA1 was predicted with high probability to be targeted to mitochondria by TargetP (score 0.671) as well as AtmtRecA (score 0.841). We determined the genomic DNA sequence encompassing PprecA1 and compared it with the cDNA sequence. The PprecA1 gene was composed of eight exons and seven introns, and the locations of the introns corresponded well with those of AtmtrecA and At2g19490 (Fig. 1B). Phylogenetic analysis of PpRecA1 and RecA homologues from other organisms revealed that PpRecA1, AtmtRecA, and At2g19490 belong to the same subgroup (Fig. 1C).

Subcellular localization of PpRecA1-GFP To determine the actual subcellular localization of PpRecA1, the first $210 \mathrm{bp}$ of the gene encompassing the coding region for the putative signal peptide was fused to the GFP gene under the control of a modified cauliflower mosaic virus $35 \mathrm{~S}$ promoter and the construct was introduced into $P$. patens protoplasts. Mitochondria stained with Mito Tracker Green FM were visualized as branched- and sausage-shapes in the protoplast (Fig. 2A). The subcellular distribution of PpRecA1-GFP was strikingly similar to
A

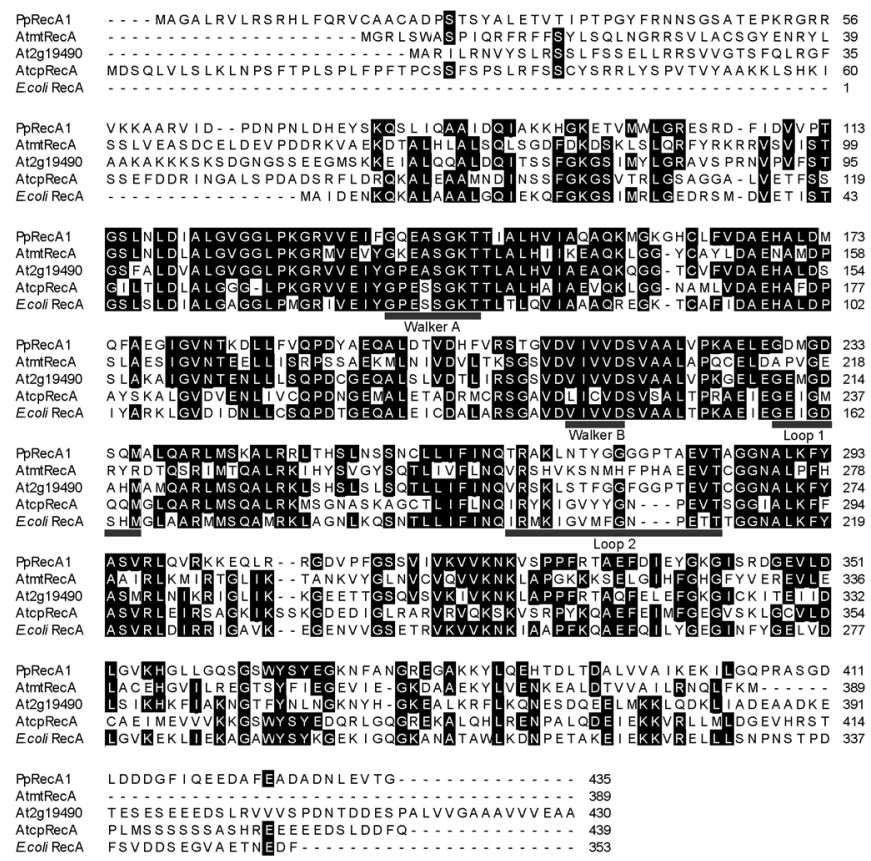

B

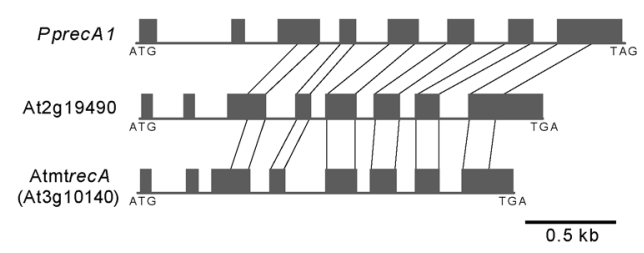

C

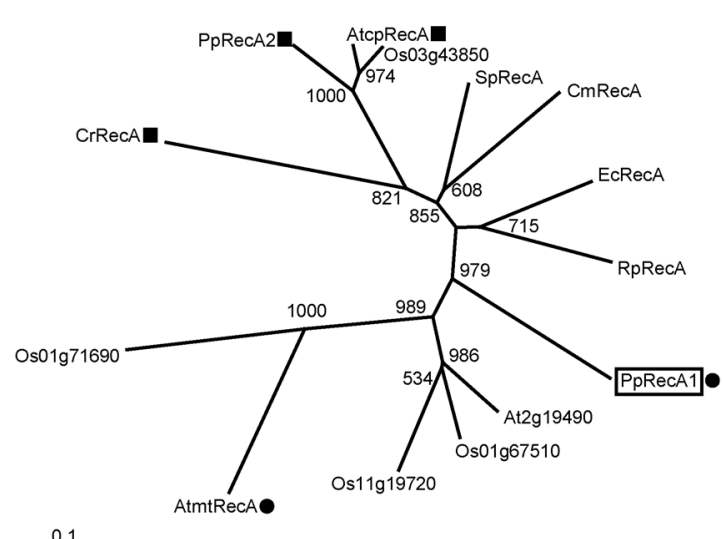

Fig. 1. (A) Alignment of the amino acid sequences of PpRecA1, three RecA homologues from A.thaliana and E. coli RecA. Sequence identities are inverted. The conserved functional domains of RecA (Lusetti and Cox, 2002) are indicated under the sequences: Walker A and B are involved in ATP hydrolysis, and loop1 and 2 are important for DNA binding. (B) Gene organizations of PprecA1, At2g19490 and AtmtrecA (At3g10140). The filled boxes and the lines between the boxes represent exons and introns, respectively. The corresponding exons are joined to each other by lines. (C) Phylogenetic relationship of PpRecA1 protein sequence to other RecA proteins. Bootstrap values are indicated at the branch points. Experimentally determined subcellular localizations are shown by closed circles (mitochondria) and closed squares (chloroplast). Ec, Eschelichia coli; Rp, Rickettsia prowazekii; Sp, Synechocystis sp. PCC 6803; Cm, Cyanidioschyzon merolae; Cr, Chlamydomonas reinhardtii; Os, Oryza sativa. Scale bar corresponds to 0.1 amino acid substitutions per residue. 


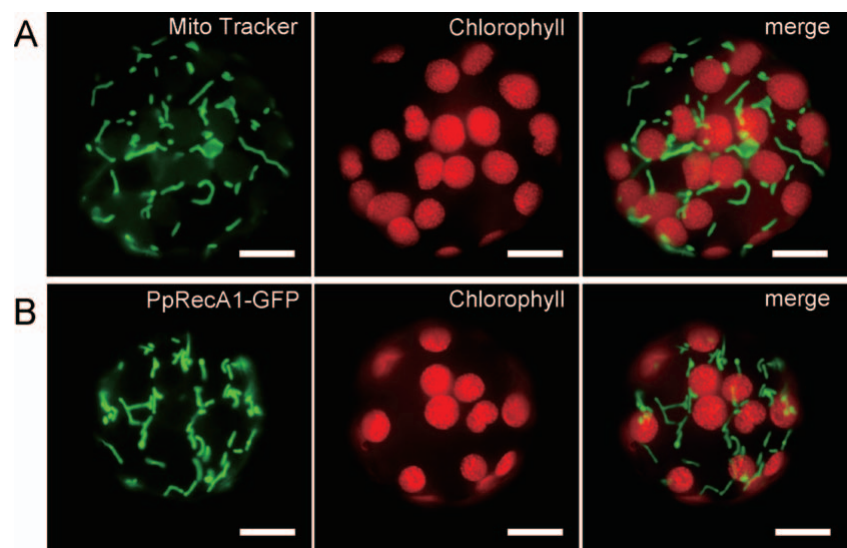

Fig. 2. Intracellular localization of PpRecA1-GFP protein. (A) Discrimination of mitochondria and chloroplasts in $P$. patens protoplast. Mitochondria were stained with Mito Tracker Green FM (green fluorescence), and chloroplasts were discriminated by chlorophyll autofluorescence (red fluorescence). Bars $=10 \mu \mathrm{m}$ (B) Intracellular localization of the targeting presequence of PpRecA1 fused to GFP, which was transiently expressed under the control of the 7133 promoter in $P$. patens protoplasts. Green fluorescence indicates GFP fused to PpRecA1.

that of mitochondria, indicating that PpRecA1 was localized to mitochondria (Fig. 2B). We confirmed that the fluorescence of the PpRecA1-GFP was co-localized to the mitochondria stained with Mito Tracker Orange (data not shown). Using this construct, we could not detect the fluorescence of the PpRecA1-GFP in chloroplast (Fig. 2B). On the other hand, the fluorescence of PpRecA2GFP was detected only in chloroplasts (Odahara and Inouye, unpublished data).

Complementation of an $E$. coli recA deficient strain by PprecA1 To investigate the functional relationship between PpRecA1 and $E$. coli RecA, the coding sequence of the PprecA1 cDNA, except for the putative signal peptide, was placed under the control of the inducible arabinose promoter and the construct was transformed into an E. coli $\mathrm{rec} A$ deficient strain. Survival rates were measured after treating the cells with the DNA crosslinking agent mitomycin $\mathrm{C}$. Upon the addition of arabinose, the surviving fraction of $E$. coli recA deficient cells bearing the PprecA1 plasmid increased by up to 10-fold compared to that of the strain bearing a plasmid lacking PprecA1, whereas no increase was observed when PprecA1 expression was repressed by the addition of glucose (Fig. 3 ). These results indicate that PpRecA1 partially complements the function of $E$. coli RecA.

Enhanced expression of PprecA1 after DNA damage Expression of E. coli recA is induced in response to DNA damage as part of the SOS response (Kowalczykowski et al., 1994). The recA homologues of pea and C. reinhardtii chloroplasts have also been suggested to increase after DNA damage (Cerutti et al., 1992, Nakazato et al.,

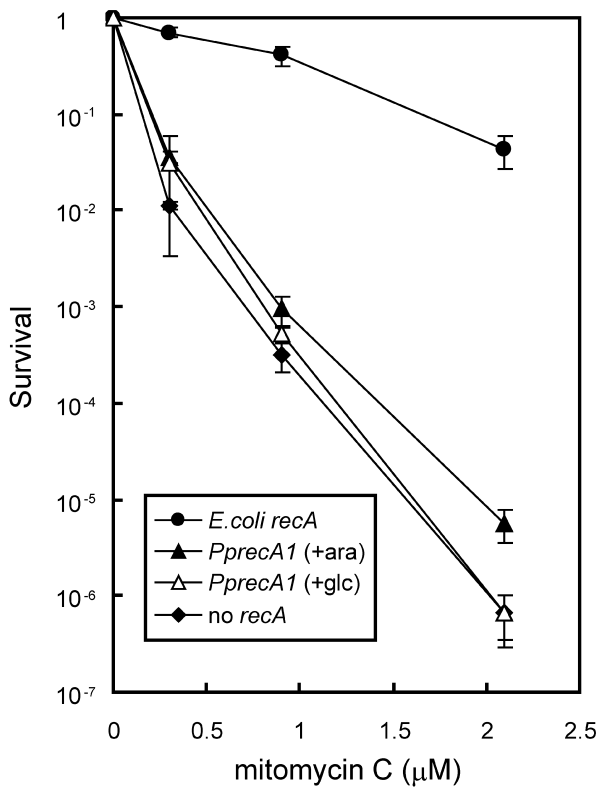

Fig. 3. Partial complementation of an E. coli recA deficient strain by PprecA1. The surviving fraction after mitomycin $\mathrm{C}$ treatment of the CSR603 strain $\left[\mathrm{recA}^{-}\right]$harboring pMAK132 with $E$. coli recA (circle), pMAK131 with PprecA1 (triangle), or pBAD24 (square) alone was calculated as described in Materials and Methods. The strain harboring pMAK131 was grown in the medium containing $0.2 \%$ arabinose (closed triangle) or $0.2 \%$ glucose (open triangle). The other strains were grown in the medium containing $0.2 \%$ arabinose. Data are expressed as mean \pm S.D. $(n=3)$.

2003). To analyze the control of mitochondrial PprecA1 gene expression in the DNA-damaged cells of $P$. patens, we analyzed the level of PprecA1 expression in MMS- or bleomycin- treated cells by semi-quantitative RT-PCR. MMS, a DNA methylating agent, is suggested to cause double strand breaks (DSBs) by forming methylated bases in DNA (Nowosielska et al., 2006), while bleomycin, a radiomimetic DNA-damaging agent, causes DSBs directly (Cramer and Painter, 1981). Total RNA was extracted from protonemata treated with MMS or bleomycin for $6 \mathrm{hr}$, and semi-quantitative RT-PCR was performed as described in Materials and Methods. PprecA1 expression was strongly induced by treatment with MMS as well as with bleomycin (Fig. 4), suggesting PprecA1 involvement in DNA repair.

Generation of PprecA1 disruptants To examine the in vivo role of PpRecA1, PprecA1 gene disruptants were generated by homologous recombination using a targeting construct in which the complete coding sequence of PprecA1 was replaced by the $h p t$ gene (Fig. 5A). Four strains of PprecA1 disruptants were generated. Each strain showed a very similar growth phenotype. (The detailed growth phenotypes of the disruptants will be published elsewhere.) Of the four, two strains (PprecA1-1 and PprecA1-2) were used for further analyses. To verify 


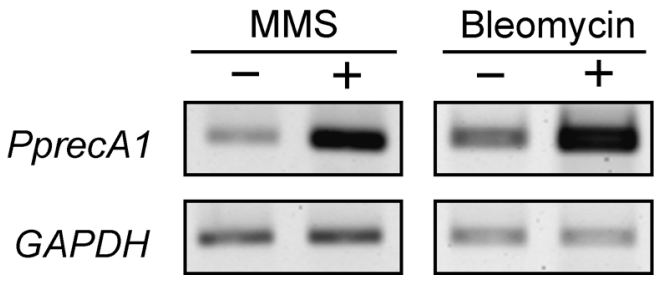

Fig. 4. Expression of PprecA1 in MMS- or bleomycin- treated cells. Total RNA was extracted from protonemata cultivated in the presence $(+)$ or absence $(-)$ of the DNA damaging agents, MMS $(20 \mathrm{mM})$ and bleomycin $(25 \mu \mathrm{g} / \mathrm{ml})$. The GAPDH gene was amplified as an internal control. PCR was performed with 28 cycles for the amplification of PprecA1 and 22 cycles for the amplification of $G A P D H$.
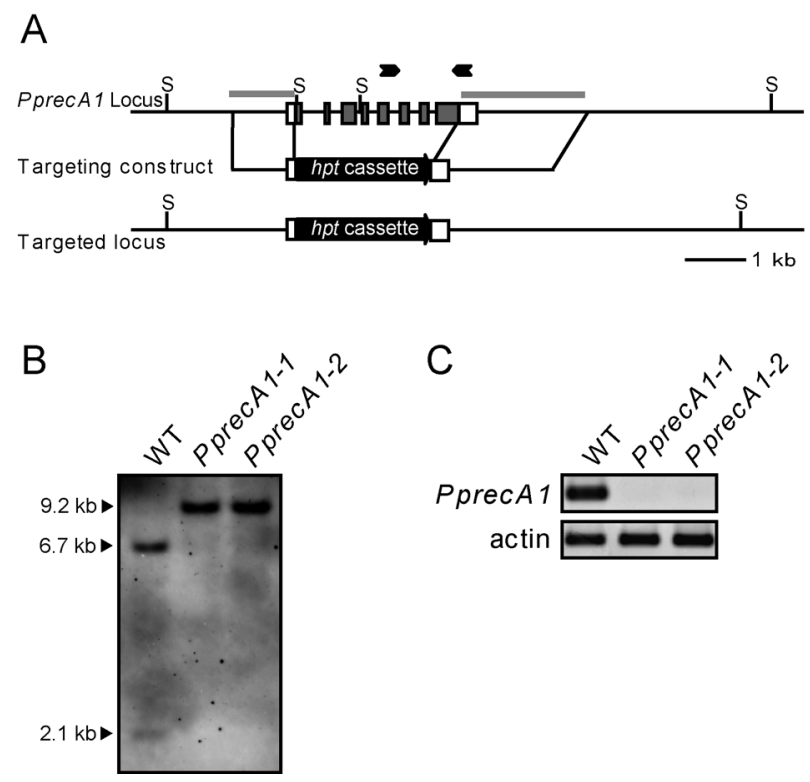

Fig. 5. Generation of PprecA1 disruptants. (A) A scheme for the disruption of the PprecA1 locus. The boxes represent exons, and the lines between boxes represent introns and noncoding flanking sequences. The coding regions are shaded in gray. The recognition sites of $S a c I$ are indicated by (S). The locations of the probes and the primers used in (B) and (C) are shown by bold gray lines and arrowheads, respectively. hpt cassette, cauliflower mosaic virus $35 \mathrm{~S}$ promoter-driven hygromycin phosphotransferase gene. (B) DNA gel blot analysis of two strains of the PprecA1 disruptants (PprecA1-1 and PprecA12). Total genomic DNA was digested with $S a c$ I. The lengths of the bands are indicated in the left side of the figure. WT, wild type. (C) Semi-quantitative RT-PCR analysis of the PprecA1 transcript in the PprecA1 disruptants. Total RNA (1 $\mu \mathrm{g})$ was reverse-transcribed, and subsequent PCR was performed with 30 cycles for PprecA1 and 22 cycles for the actin gene, which was used as an internal control.

the proper integration of the hpt gene cassette into the chromosomal PprecA1 locus, DNA gel blot analysis was performed using a mixture of two probes that hybridized to the upstream region and downstream region of genomic PprecA1 (Fig. 5B). Two bands of $6.7-\mathrm{kb}$ and $2.1-\mathrm{kb}$ bands were generated from the PprecA1 locus of the WT strain. When we used the genomic DNA from either one of the two PprecA1 disruptants, the two bands disappeared and were replaced by a $9.2-\mathrm{kb}$ band. This indicated that one copy of the targeting construct containing the $h p t$ gene cassette $(2.0 \mathrm{~kb})$, which has no $S a c \mathrm{I}$ restriction site, had replaced the PprecA1 coding region $(2.7 \mathrm{~kb})$ which has two $S a c$ I sites. The disappearance of the PprecA1 transcript was confirmed by semi-quantitative RT-PCR (Fig. 5C).

Recovery of mitochondrial DNA from MMS damage in the PprecA1 disruptants MMS is a DNA alkylating agent that produces predominantly N-methylated bases in DNA (Pegg, 1984). In Salmonella typhimurium, mutations in the recA gene sensitize cells to MMS (Shanabruch et al., 1983). It has been reported that RecA and other recombination factors are needed to repair MMS-damaged DNA (Nowosielska et al., 2006). We therefore analyzed the repair of MMS-damaged mtDNA in a PprecA1 disruptant by using a quantitative PCR assay, whose amplification efficiency can be severely reduced by DNA lesions that block progression of the Taq-derived polymerase. Thus, reduced amplification in this assay can be taken as a measure of the amount of damaged DNA template (Govan et al., 1990).

After treatment of protonemata with MMS, genomic DNA was extracted and used as a template for PCR, which resulted in the amplification of a $3.2 \mathrm{~kb}$ segment of the mitochondrial encoded cox 2 gene and a $3.9 \mathrm{~kb}$ segment of the nuclear encoded PpMADS2 gene. First we confirmed that PCR amplification of both DNA segments decreased with increasing MMS concentration (data not shown), indicating that the PCR assay was proficient for monitoring the level of MMS-damaged DNA. Next, we examined the time course of amplification to compare the recovery of DNA from MMS damage in the PprecA1 disruptant to that in the WT. To do this, protonemata were cultured in MMS-free growth medium for different time periods so that DNA repair could occur following treatment with MMS. Subsequently, genomic DNA was extracted and the PCR assay was carried out. The amplification of damaged segment was normalized to the amplification of the nondamaged control, which gave a relative amplification ratio. As shown in Fig. 6, the amplification of cox 2 and PpMADS2 increased with time, indicating the repair of DNA damage caused by MMS in mitochondrial DNA as well as in nuclear DNA. There was no significant difference in the amplification of the PpMADS2 locus between the WT and the PprecA1 disruptant (Fig. 6A). However, the amplification of the cox2 locus of the disruptant cells clearly declined over time compared to the amplification of the same locus of WT cells (Fig. 6B). We also observed a similar decline in the amplification of two other loci in mtDNA, nad7 and atp9, but no decline in the amplification of nuclear encoded 
A

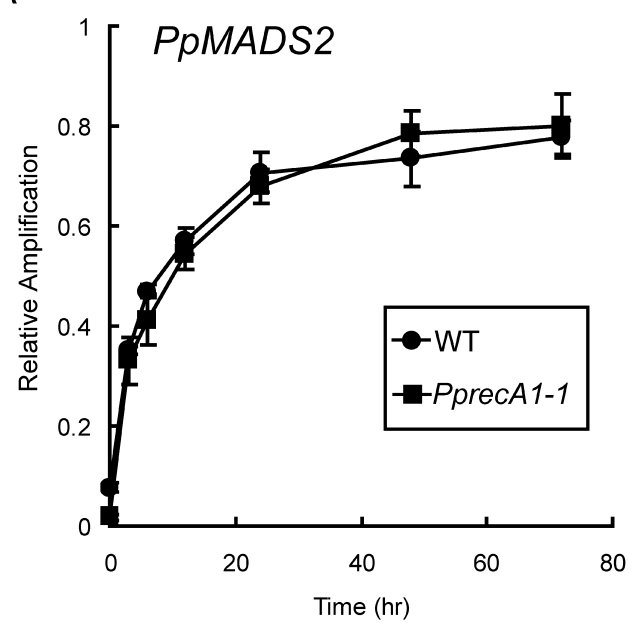

B

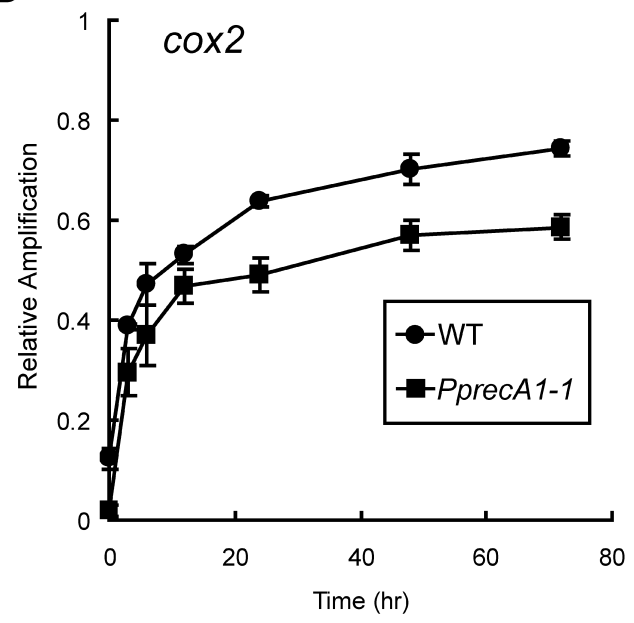

Fig. 6. Quantitative PCR assay for monitoring DNA repair activity. Protonemata of the WT and one of the PprecA1 disruptants (PprecA1-1) were exposed to $20 \mathrm{mM}$ of MMS for 60 min, and then were either harvested immediately or allowed to recover in growth medium before extracting total genomic DNA. The PCR assay was performed as described in Materials and Methods to amplify the PpMADS2 gene for nuclear DNA (panel A) and the cox2 gene for mitochondrial DNA (panel B). Relative amplification represents the amount of PCR product from a damaged DNA template divided by the amount of PCR product from a nondamaged template. Circle, WT; square, PprecA1-1. Error bars indicate standard errors of the mean for three independent experiments.

GAPDH and tubG loci (data not shown) in the PprecA1 disruptants. These results suggest that the PprecA1 disruptant is defective in the repair of DNA damaged directly or indirectly by MMS.

\section{DISCUSSION}

We have investigated the role of mitochondrial recA (PprecA1) in the repair of damaged mtDNA in P. patens. To examine the role of PprecA1, we generated mutants

with complete deletions of PprecA1, which, to our knowledge, is the first time a bacterial-type $r e c A$ gene has been mutated in a plant. By analyzing the mutant, we found that the recovery of mtDNA from MMS damage was inefficient in the PprecA1 disruptant. This result together with the partial complementation of a recA deletion mutation in $E$. coli by PprecA1 and its remarkable induction by DNA damage suggests that PprecA1 participates in the repair of mtDNA.

Plant homologues of bacterial-type RecA were divided into two groups by phylogenetic analysis (see Fig. 1C). One group contains mitochondrial-targeted RecA proteins, including PpRecA1 identified in this report, and AtmtRecA (Khazi et al., 2003), suggesting that other members of this group are also mitochondrial-targeted. Another group contains chloroplast-targeted RecA proteins, including CrRecA (Nakazato et al., 2003), AtcpRecA (Cerutti et al., 1992) and PpRecA2 (Odahara and Inouye, unpublished results), suggesting that other members of this group are also chloroplast-targeted. Our phylogenetic analysis shows a relationship between plant mitochondrial RecA and proteobacterial RecA and between chloroplast RecA and cyanobacterial RecA (see Fig. 1C), suggesting that mitochondrial and chloroplast RecA originated from proteobacteria and cyanobacteria, respectively. Consistently, Lin et al. (2006) has discussed the origin of plant bacterial RecA.

Phylogenetic analysis suggests that each of C. reinhardtii (CrRecA) and C. merolae (CmRecA) has a single chloroplast-targeted RecA protein (see Fig. 1C), and indeed, CrRecA has been reported to be localized to the chloroplast (Nakazato et al., 2003). These findings suggest that mitochondrial recA is absent in C. merolae as well as in $C$. reinhardtii, although we have to be careful for the incompleteness of the $C$. reinhardtii genome sequence. Homologous recombination has been reported in $C$. reinhardtii mitochondria (Yamasaki et al., 2005). Thus, a RecA-independent recombination system that is distinct from the RecA-dependent system in P. patens and other land plant mitochondria may be operative in these algal mitochondria.

As presented in this study, PprecA1 complemented an E. coli recA deficient strain. This suggests that PpRecA1 has the ability to repair damaged DNA, like E. coli RecA. However, the degree of complementation was low as compared to that of $E$. coli RecA (see Fig. 3). E. coli RecA, which plays a central role in recombinational repair pathways, is also involved in the SOS response, which also contributes to DNA repair (Roca and Cox, 1997). This could suggest that the greater survival rate of the recA deficient strain transformed with the recA expression plasmid might be due to the SOS response. The presence of an SOS response in plant mitochondria is unclear, and we were unable to find a LexA homologue in the plant genomes (data not shown), suggesting that PpRecA1 
would be unable to induce the SOS response in E. coli. Furthermore, for efficient recombination, interaction between RecA and many other recombinational factors (e.g. RecBCD; Spies and Kowalczykowski, 2006) is required in E. coli. PpRecA1 may fail to interact with such $E$. coli recombinational factors, since no obvious homologues of these recombination factors has been found in the plant genomes (data not shown). It is also possible that PpRecA1 lost some function in the course of evolution from its proteobacterial counterpart. Indeed, there is a clear difference in the amino acid sequence of DNAbinding domain Loop2 between plant mitochondrial RecA proteins (PpRecA1, AtmtRecA and At2g19490) and proteobacterial RecA (see Fig. 1A). In addition, insufficiency of PprecA1 product might have caused the decrease in the degree of complementation, as translation efficiency might be reduced because of the minor codon usage for $E$. coli in PprecA1.

Our results showing that PprecA1 is induced by DNA damage are consistent with a role for PprecA1 in DNA repair. Although we assumed that PprecA1 was induced by mtDNA damage, we cannot state with certainty that this was the primary cause, because nuclear and chloroplast DNAs would also have been damaged in our assay. Specific damages to mtDNA will be required to elucidate the regulatory mechanism that responds to the DNA damage.

Using the quantitative PCR assay, we observed enhanced amplification of nuclear and mitochondrial DNA after the WT strain was allowed to recover from MMSinduced damage. If the MMS damage was completely removed, the relative amplification, which is a ratio of the amount of amplified product from damaged DNA to that from nondamaged DNA, would be 1 . However, the amplification ratio was almost saturated to $0.7 \sim 0.8$, even in the case of the WT strain (see Fig. 6). Microscopic observation of the MMS-treated cells revealed that some of the cells, especially the apical cells, were dead (data not shown). The incomplete recovery from DNA damage, as measured in the DNA amplification, may be caused by the unrepaired and/or degraded DNA in these dead cells.

In the PprecA1 disruptant, we found that the recovery of mtDNA from MMS damage, as measured by the DNAamplification assay, was clearly lower than that of WT. This indicates residual damages on mtDNA and/or decrease in the copy number of mtDNA in the PprecA1 disruptant. In the first $\sim 12 \mathrm{hr}$ period of cultivation of the MMS-treated PprecA1 disruptants, however, rapid recovery of DNA, as measured by the increase in the efficiency of mtDNA amplification, was nonetheless observed (see Fig. 6B). In E. coli, MMS-damaged bases are repaired by base excision repair (BER), the direct removal of methyl groups or oxidative demethylation (Nowosielska et al., 2006). Similar repair system(s), which are independent of RecA, might be responsible for this repair in the
PprecA1 mutant. Besides such repair systems removing MMS damage, homologous recombination is required to reduce MMS toxicity in $E$. coli (Nowosielska et al., 2006). A possible reason for this is that homologous recombination is essential for the repair of DSBs and single-strand gaps produced by stalled DNA replication forks or singlestrand nicks introduced by AP-endonuclease action in BER (Nowosielska et al., 2006). Accordingly, the lower rate of the recovery of mtDNA from MMS damage in the PprecA1 disruptants suggests the presence of unrepaired DSBs and single-strand gaps on the mtDNA and subsequent decrease in the copy number of the mtDNA due to the genomic instability. PpRecA1 may play an important role in homologous recombination to repair such DSBs and single-strand gaps on the mtDNA.

In plant mitochondria, reactive oxygen species (ROS) are produced (Sweetlove and Foyer, 2004) and thought to cause oxidative damage. Generally, most of the oxidative damage caused by ROS is repaired by BER (Seeberg et al., 1995), in the course of BER, DSBs and singlestrand gaps can be generated. We suggest that PpRecA1 may play an important role in the repair of such DNA lesions in plant mtDNA.

We are grateful to K. Fujiwara and M. Umeda for technical assistance. We are also grateful to T. Nishiyama and S. Aoki for providing the plasmids pTN186 and p7133TP-sGFP. This work was supported by a Grant-in-Aid for Japan Society for the Promotion of Science Fellows 08575 (to M. O.) and a Grant-inAid for Scientific Research on Priority Areas (to T. F. and M. H.) from the Ministry of Education, Culture, Sports, Science and Technology of Japan, and partially supported by the Frontier Project 'Environmental Changes and Life Adaptation strategies' (to Y. S.).

\section{REFERENCES}

Bishop, D. K., Park, D., Xu, L., and Kleckner, N. (1992) DMC1: a meiosis-specific yeast homolog of $E$. coli recA required for recombination, synaptonemal complex formation, and cell cycle progression. Cell 69, 439-456.

Cao, J., Combs, C., and Jagendorf, A. T. (1997) The chloroplastlocated homolog of bacterial DNA recombinase. Plant Cell Physiol. 38, 1319-1325.

Cerutti, H., and Jagendorf, A. T. (1993) DNA Strand-Transfer Activity in Pea (Pisum sativum L.) Chloroplasts. Plant Physiol. 102, 145-153.

Cerutti, H., Osman, M., Grandoni, P., and Jagendorf, A. T. (1992) A homolog of Escherichia coli RecA protein in plastids of higher plants. Proc. Natl. Acad. Sci. USA 89, 8068-8072.

Cove, D., Bezanilla, M., Harries, P., and Quatrano, R. (2006) Mosses as Model Systems for the Study of Metabolism and Development. Annu. Rev. Plant Biol. 57, 497-520.

Cramer, P., and Painter, R. B. (1981) Bleomycin-resistant DNA synthesis in ataxia telangiectasia cells. Nature 291, 671672.

Emanuelsson, O., Nielsen, H., Brunak, S., and von Heijne, G. (2000) Predicting subcellular localization of proteins based on their N-terminal amino acid sequence. J. Mol. Biol. 300, 1005-1016.

Govan, H. L. 3rd, Valles-Ayoub, Y., and Braun, J. (1990) Fine- 
mapping of DNA damage and repair in specific genomic segments. Nucleic Acids Res. 18, 3823-3830.

Guzman, L. M., Belin, D., Carson, M. J., and Beckwith, J. (1995) Tight regulation, modulation, and high-level expression by vectors containing the arabinose PBAD promoter. J. Bacteriol. 177, 4121-4130.

Hara, K., Morita, M., Takahashi, R., Sugita, M., Kato, S., and Aoki, S. (2001a) Characterization of two genes, Sig1 and Sig2, encoding distinct plastid sigma factors(1) in the moss Physcomitrella patens: phylogenetic relationships to plastid sigma factors in higher plants. FEBS Lett. 499, 87-91.

Hara, K., Sugita, M., and Aoki, S. (2001b) Cloning and characterization of the cDNA for a plastid sigma factor from the moss Physcomitrella patens. Biochim. Biophys. Acta 1517, 302306.

Henschel, K., Kofuji, R., Hasebe, M., Saedler, H., Muenster, T., and Theissen, G. (2002) Two ancient classes of MIKC-type MADS-box genes are present in the moss Physcomitrella patens. Mol. Biol. Evol. 19, 801-814.

Khazi, F. R., Edmondson, A. C., and Nielsen, B. L. (2003) An Arabidopsis homologue of bacterial RecA that complements an $E$. coli recA deletion is targeted to plant mitochondria. Mol. Genet. Genomics 269, 454-463.

Kowalczykowski, S. C., Dixon, D. A., Eggleston, A. K., Lauder, S. D., and Rehrauer, W. M. (1994) Biochemistry of homologous recombination in Escherichia coli. Microbiol. Rev. 58, 401-465.

Leech, M. J., Kammerer, W., Cove, D. J., Martin, C., and Wang, T. L. (1993) Expression of myb-related genes in the moss, Physcomitrella patens. Plant J. 3, 51-61.

Lin, Z., Kong, H., Nei, M., and Ma, H. (2006) Origins and evolution of the recA/RAD51 gene family: Evidence for ancient gene duplication and endosymbiotic gene transfer. Proc. Natl. Acad. Sci. USA 103, 10328-10333.

Lusetti, S. L., and Cox, M. M. (2002) The bacterial RecA protein and the recombinational DNA repair of stalled replication forks. Annu. Rev. Biochem. 71, 71-100.

Manchekar, M., Scissum-Gunn, K., Song, D., Khazi, F., McLean, S. L., and Nielsen, B. L. (2006) DNA recombination activity in soybean mitochondria. J. Mol. Biol. 356, 288-299.

Matsuzaki, M., Misumi, O., Shin, I. T., Maruyama, S., Takahara, M., et al. (2004) Genome sequence of the ultrasmall unicellular red alga Cyanidioschyzon merolae 10D. Nature 428, 653-657.

Mitsuhara, I., Ugaki, M., Hirochika, H., Ohshima, M., Murakami, T., et al. (1996) Efficient promoter cassettes for enhanced expression of foreign genes in dicotyledonous and monocotyledonous plants. Plant Cell Physiol. 37, 49-59.

Murray, M. G., and Thompson, W. F. (1980) Rapid isolation of high molecular weight plant DNA. Nucleic Acids Res. 8, 4321-4325.

Nakazato, E., Fukuzawa, H., Tabata, S., Takahashi, H., and Tanaka, K. (2003) Identification and expression analysis of cDNA encoding a chloroplast recombination protein REC1, the chloroplast RecA homologue in Chlamydomonas reinhardtii. Biosci. Biotechnol. Biochem. 67, 2608-2613.

Newton, K. J., Gabay-Laughnan, S., and Paepe, R. D. (2004) Mitochondrial Mutation in Plants. In: Plant Mitochondria (eds.: Day, D. A., Millar, A. H. and Whelan, J.), pp. 121-141. Kluwer Academic Publishers, Dordrecht.

Nishiyama, T., Hiwatashi, Y., Sakakibara, I., Kato, M., and Hasebe, M. (2000) Tagged mutagenesis and gene-trap in the moss, Physcomitrella patens by shuttle mutagenesis. DNA Res. 7, 9-17.

Nishiyama, T., Fujita, T., Shin, I. T., Seki, M., Nishide, H., et al. (2003) Comparative genomics of Physcomitrella patens gametophytic transcriptome and Arabidopsis thaliana: implication for land plant evolution. Proc. Natl. Acad. Sci. USA 100, 8007-8012.

Nowosielska, A., Smith, S. A., Engelward, B. P., and Marinus, M. G. (2006) Homologous recombination prevents methylation-induced toxicity in Escherichia coli. Nucleic Acids Res. 34, 2258-2268.

Palmer, J. D., Adams, K. L., Cho, Y., Parkinson, C. L., Qiu, Y. L., and Song, K. (2000) Dynamic evolution of plant mitochondrial genomes: mobile genes and introns and highly variable mutation rates. Proc. Natl. Acad. Sci. USA 97, 6960-6966.

Pegg, A. E. (1984) Methylation of the O6 position of guanine in DNA is the most likely initiating event in carcinogenesis by methylating agents. Cancer Invest. 2, 223-231.

Roca, A. I., and Cox, M. M. (1997) RecA protein: structure, function, and role in recombinational DNA repair. Prog. Nucleic Acid Res. Mol. Biol. 56, 129-223.

Saitou, N., and Nei, M. (1987) The neighbor-joining method; a new method for reconstructing phylogenetic trees. Mol. Biol. Evol. 4, 406-425.

Sancar, A., and Rupert, C. S. (1978) Determination of plasmid molecular weights from ultraviolet sensitivities. Nature 272, 471-472.

Schaefer, D. G., and Zryd, J. P. (2001) The moss Physcomitrella patens, now and then. Plant Physiol. 127, 1430-1438.

Seeberg, E., Eide, L., and Bjoras, M. (1995) The base excision repair pathway. Trends Biochem. Sci. 20, 391-397.

Shanabruch, W. G., Rein, R. P., Behlau, I., and Walker, G. C. (1983) Mutagenesis, by methylating and ethylating agents, in mutH, mutL, mutS, and uvrD mutants of Salmonella typhimurium LT2. J. Bacteriol. 153, 33-44.

Shinohara, A., Ogawa, H., and Ogawa, T. (1992) Rad51 protein involved in repair and recombination in $S$. cerevisiae is a RecA-like protein. Cell 69, 457-470.

Spies, M., and Kowalczykowski, S. C. (2006) The RecA binding locus of RecBCD is a general domain for recruitment of DNA strand exchange proteins. Mol. Cell 21, 573-580.

Sweetlove, L. J., and Foyer, C. H. (2004) Roles for Reactive Oxygen Species and Antioxidants in Plant Mitochondria. In: Plant Mitochondria (eds.: Day, D. A., Millar, A. H., and Whelan, J.), pp. 307-320. Kluwer Academic Publishers, Dordrecht.

The Arabidopsis Genome Initiative (2000) Analysis of the genome sequence of the flowering plant Arabidopsis thaliana. Nature 408, 796-815.

Thompson, J. D., Higgins, D. G., and Gibson, T. J. (1994) CLUSTAL W: improving the sensitivity of progressive multiple sequence alignment through sequence weighting, position-specific gap penalties and weight matrix choice. Nucleic Acids Res. 22, 4673-4680.

Unseld, M., Marienfeld, J. R., Brandt, P., and Brennicke, A. (1997) The mitochondrial genome of Arabidopsis thaliana contains 57 genes in 366,924 nucleotides. Nat. Genet. 15, $57-61$.

Yamasaki, T., Kurokawa, S., Watanabe, K. I., Ikuta, K., and Ohama, T. (2005) Shared molecular characteristics of successfully transformed mitochondrial genomes in Chlamydomonas reinhardtii. Plant Mol. Biol. 58, 515-527. 